\title{
The Heterogeneity of the Cigarette Price Effect on Body Mass Index
}

\author{
George L. Wehby ${ }^{*}$ and Charles J. Courtemanche ${ }^{\dagger}$ \\ George L. Wehby: george-wehby@uiowa.edu; Charles J. Courtemanche: ccourtemanche@gsu.edu \\ "Department of Health Management and Policy, College of Public Health, University of lowa, 105 \\ River St., N248 CPHB, lowa City, IA 52242. Phone: 319- 384-3814 \\ tDepartment of Economics, Andrew Young School of Policy Studies, Georgia State University, \\ Atlanta, GA 30303 and National Bureau of Economic Research, Cambridge, MA. Phone: \\ 404-413-0141
}

\begin{abstract}
Previous studies estimate the average effect of cigarette price on body mass index (BMI), with recent research showing that their different methodologies all point to a negative effect after several years. This literature, however, ignores the possibility that the effect could vary throughout the BMI distribution or across socioeconomic and demographic groups due to differences in underlying obesity risks or preferences for health. We evaluate heterogeneity in the long-run impact of cigarette price on BMI by performing quantile regressions and stratifying the sample by race, education, age, and sex. Cigarette price has a highly heterogeneous negative effect that is more than three times as strong at high BMI levels - where weight loss is most beneficial for health - than at low levels. The effects are also strongest for blacks, college graduates, middleaged adults, and women. We also assess the implications for disparities, conduct robustness checks, and evaluate potential mechanisms.
\end{abstract}

\section{Keywords}

obesity; body mass index; racial disparities; smoking; cigarette prices; cigarette taxes; quantile regression; fruit; vegetables; exercise; physical activity

\section{Introduction}

Obesity has become one of the leading population-level health risks in the United States.

Not only is the prevalence high, but obesity rates have increased significantly and steadily in recent decades, from $15 \%$ in the early 1970 s to $34 \%$ in $2007-2008$ (CDC, 2010). Obesityrelated illnesses such as heart disease and diabetes now lead to an estimated 112,000 deaths and $\$ 168.4$ billion in medical expenditures per year (Cawley and Meyerhoefer, 2010; Flegal et al., 2010; Sturm, 2002). ${ }^{1}$ This has prompted a major research effort to explain the rising incidence of obesity, including an investigation of whether changes in other health characteristics or behaviors may have played a role.

The most notable population-level health behavior change concurrent with the rise in obesity was a dramatic decline in cigarette smoking. Between 1965 and 2007, the smoking rate

Correspondence to: George L. Wehby, george-wehby@uiowa . edu.

${ }^{1}$ An individual is considered obese if her body mass index (BMI), or weight in kilograms divided by height in squared meters, is greater than or equal to 30 . 
among adults in the US decreased from $42 \%$ to $20 \%$ (CDC, 2010). The simultaneous nature of the trends in smoking and obesity raises the question of whether the two are causally related. This question is further motivated by the potential biologic pathways through which smoking could affect body weight. Smoking may reduce appetite and enhance metabolism, leading to lower caloric intake and higher caloric expenditure (Pinkowish, 1999). This theory is supported by mice studies that show reductions in appetite, weight, and fat storage as well as biochemical changes in the brain with nicotine exposure (Chen et al., 2008; Chen et al., 2005). However, other factors suggest the opposite or no effect of smoking on obesity. Smoking may reduce the ability to exercise by impairing respiratory functioning (Hedenstrom et al., 1986). Also, quitting smoking could lead to healthier eating and exercise decisions by affecting expected longevity or general enthusiasm about health (Courtemanche, 2009). Furthermore, the drop in smoking is unlikely to explain the rise in childhood obesity unless changes in weight among adults caused by the decrease in smoking influence weight in children.

Motivated by this theoretical ambiguity, several recent economic studies have attempted to determine the causal effect of cigarette costs on weight. ${ }^{2}$ Chou et al. $(2002 ; 2004)$ estimate positive relationships between state cigarette price and both body mass index and obesity using the Behavioral Risk Factor Surveillance System (BRFSS) and models that include state fixed effects, a quadratic time trend (or no time trend), and a set of control variables. Gruber and Frakes (2006) show that replacing cigarette price with state cigarette tax rate and the quadratic time trend with time period fixed effects switches the sign of the effect to negative. Rashad et al. (2006) use a methodology similar to Gruber and Frakes but a different dataset - the National Health and Nutrition Examination Survey (NHANES) - and estimate a small positive effect of cigarette taxes on weight. Nonnemaker et al. (2009) use the BRFSS and find that including linear state-specific time trends leads to the estimation of a small and insignificant relationship between cigarette costs and weight across the population using either price or tax as an instrument for price. Baum (2009) considers the National Longitudinal Survey of Youth (NLSY) and a difference-in-difference approach where the "treatment" group consists of individuals who have smoked at least 100 cigarettes in their life and are therefore most likely to be responsive to cigarette costs. He estimates a positive effect of cigarette costs on weight regardless of whether the other features of the model are similar to those of Chou et al. (2002; 2004) or Gruber and Frakes (2006). Most recently, Courtemanche (2009) notes that the aforementioned papers focus on the impact of contemporaneous cigarette costs, which may be insufficient to capture the entire long-run effect since all three stages of the relationship - the effect of prices on smoking, the effect of smoking on behaviors, and the effect of behaviors on BMI - could occur gradually. He uses both the NLSY and BRFSS and shows that all the different methodologies used in the literature point to a negative long-run effect of cigarette costs on weight if the effect is allowed to occur slowly over a six year period. ${ }^{3}$

A limitation common to all of these studies is a focus on the effects of cigarette costs on the mean of BMI or obesity status. While informative, focusing on "mean effects" may mask substantial heterogeneity in the effects of cigarette costs across the population. This heterogeneity may result from differences in biologic, demographic, socioeconomic, and environmental factors as well as in preferences for body weight. Evaluating if cigarette costs have heterogeneous effects is necessary to understand changes in the entire BMI distribution - as opposed to merely one moment of the distribution - and also to identify the groups that are most affected by these changes. This has important implications for understanding how

\footnotetext{
${ }_{3}^{2}$ See Courtemanche (2009) for a more detailed description of the models used by the different papers in the literature.

${ }^{3}$ Other related research shows that overweight or obese female adolescents are more likely to initiate smoking, perhaps as a method of weight control (Cawley et al., 2004; Rees and Sabia, 2010).
} 
cigarette price changes over the past three decades have influenced the disparities in weight across the BMI distribution and between various demographic and socioeconomic groups.

While heterogeneity by observed characteristics can be easily evaluated by stratifying the study sample, much of the heterogeneity may result from unobserved characteristics. Studies typically find that observable individual characteristics and area-level economic factors explain only a small portion of the variation in body weight. ${ }^{4}$ Given that most of the determinants of obesity are still generally unknown, identifying the heterogeneity due to unobserved characteristics is critical to our understanding of how different types of people respond differently to changing cigarette costs. In this study, we identify the heterogeneity in the long-run effect of cigarette price on BMI due to unobservable characteristics by using quantile regression, a semi-parametric technique that estimates the impacts at different locations along the BMI distribution. We conduct quantile regressions for the total sample as well as for subsamples stratified by race, education, age, and sex, observable characteristics that have been shown to influence BMI. To our knowledge this is the first paper to study the effects of cigarette costs on body weight using quantile regression and to examine heterogeneity on the bases of education or age.

Our quantile regressions provide evidence that the long-run effect of cigarette price on BMI, while consistently negative, is more than three times as strong at high levels of BMI than at low levels. The health benefits - and corresponding savings in medical expenses - from the reduction in population weight due to higher cigarette prices are therefore more substantial than estimates of mean effects would suggest. The subsample analyses show that the mean effects and the effects at most of the quantiles are strongest for blacks, college graduates, middle-aged adults, and females. We then test the implications of these results for disparities, finding that rising cigarette prices have reduced the obesity or severe obesity (BMI 235 ) disparities between blacks and whites, young and middle-aged adults, and women and men, but increased disparities between those without a high school degree and college graduates and between young and older adults. Next, we conduct a number of robustness checks and find that the key results are generally not sensitive to specification. Finally, we investigate how the influences of cigarette prices on smoking and obesity-related behaviors vary by BMI. The long-run effect of cigarette price on cigarettes smoked is strongest at the tails of the BMI distribution, a pattern that mirrors its effect on exercise. In contrast, cigarette prices have little to no impact on the variables related to food and alcohol consumption at any BMI level. We hypothesize that the heterogeneous effects of cigarette prices on exercise combined with heterogeneous effects of exercise on BMI may help to explain the variation in the impact of cigarette price on BMI across the BMI distribution.

\section{Data}

The literature on the effect of cigarette cost on body weight utilizes three different individual-level datasets: the pooled cross-sectional BRFSS (Chou et al., 2002; Chou et al., 2004; Courtemanche, 2009; Gruber and Frakes, 2006; Nonnemaker et al., 2009), the pooled cross-sectional NHANES (Rashad et al, 2006), and the panel NLSY (Baum, 2009;

Courtemanche, 2009). The advantage of the BRFSS is its enormous sample size, which numbers in the millions as opposed to the tens-of-thousands for the NHANES and NLSY. A large sample is necessary for the precise estimation of the impacts of aggregate-level

\footnotetext{
${ }^{4}$ For example, the $\mathrm{R}^{2}$ of the models for BMI and obesity in Chou et al. (2004), which included several demographic and economic factors, is less than 0.1 . The degree to which the unobservable determinants of obesity are environmental as opposed to genetic is the subject of debate. Twin studies provide evidence of a high genetic heritability of about $80 \%$ for body mass index (BMI) and $73 \%$ for obesity (Hjelmborg et al., 2008; Watson et al., 2006). Some genes, such as FTO (Dina et al., 2007; Frayling et al., 2007; Kring et al., 2008; Scuteri et al., 2007; Villalobos-Comparan et al., 2008) and MCR4 (Loos et al., 2008; Qi et al., 2008; Willer et al., 2009) have been found to have consistent effects on body weight but these two genes explain a very small percentage of the weight variation.
} 
variables such as state cigarette price. The advantage of the NHANES is its inclusion of actual, as opposed to merely self-reported, height and weight. The advantage of the NLSY is its panel nature, which enables the inclusion of individual (rather than just state) fixed effects and the tracking of movement between states over time, which reduces measurement error when creating lagged cigarette cost variables.

A large sample size is of paramount importance when splitting the sample into race, age, and education groups and employing quantile regression, so we utilize the BRFSS in this study. Results from the literature suggest that this decision is unlikely to lead to inappropriate conclusions. Courtemanche (2009) found that the estimated average effects were similar using both the NLSY and BRFSS but that the BRFSS estimates were more precise. The NLSY regressions' use of individual as opposed to state fixed effects and tracking of between-state moves therefore did not meaningfully impact the results. This is not surprising, as the cigarette cost variables are state-level while only a fraction of individuals switch states over a six year period. The literature also shows that results from the BRFSS are not sensitive to the correction of self-reported height and weight for measurement error. ${ }^{6}$

We utilize the 1984 to 2005 waves of the BRFSS, a telephone survey of the health conditions and risky behaviors of randomly-selected individuals conducted by state health departments and the Center for Disease Control. Only 15 states participated in 1984, but this number steadily grew to 40 by 1989 and all 50 by 1996 . The number of survey respondents also increased over time, from 12,258 in 1984 to 356,112 in 2005. We compute BMI based on the respondents' self-reported height and weight. The BRFSS also includes a variety of demographic information that we use to construct a set of individual-level control variables. These variables include age, income, and dummies for gender, race, marital status, and education.

Several other health behavior-related BRFSS variables are used in the investigation of mechanisms of the effects of cigarette prices on body weight. Our smoking variables are a dummy for whether the individual currently smokes, number of cigarettes smoked per day by smokers, and cigarettes per day for the whole sample with zeros assigned to nonsmokers. The BRFSS contains information on the frequency and duration of the respondents' two main sources of exercise; we use this to compute minutes of exercise per week (an underestimate for those with three or more sources of exercise). Although the BRFSS does not report detailed information on calorie consumption for most of the sample period, we use three proxy variables for diet healthfulness: servings of fruits and vegetables per day, a dummy for whether the respondent is restricting consumption of calories or fat in order to lose weight or maintain her current weight, and alcoholic drinks per month.

We measure cigarette costs with annual state-level cigarette prices from The Tax Burden on Tobacco (Orzechowski and Walker, 2006). These prices are for a pack of twenty cigarettes, and represent averages across single packs, cartons, and vending machine sales (Chaloupka and Grossman, 1996). They are inclusive of state and federal excise taxes but not sales taxes. After 1989, The Tax Burden on Tobacco reported prices both including and excluding generic brands. We follow Chou et al. (2004) and use the series excluding generics to allow for greater comparability across the sample period. We adjust prices for inflation using the Consumer Price Index for all urban consumers from the Bureau of Labor Statistics. The Tax

\footnotetext{
${ }^{6}$ Specifically, Cawley (1999) introduced a "correction" for self-reported BMI that involves using the NHANES to estimate actual BMI as a function of self-reported BMI. Gruber and Frakes' (2006) dataset does not employ this correction, but they show that their specification changes lead to virtually identical results using Chou et al.'s (2002 and 2004) data, which does use the correction. The conclusions reached using the BRFSS are therefore not sensitive to the utilization of the correction, so we do not use it in this paper.
} 
Burden on Tobacco also provides monthly state cigarette excise tax rates, which we use instead of price in a robustness check.

We also include state-level control variables in some regressions. Number of health clubs, golf courses, or members-only sports clubs in the state - which we add together and refer to as "fitness centers" - come from the Economic Census, conducted by the U.S. Bureau every five years. We use data from the 1987, 1992, 1997, and 2002 censuses and interpolate for the other years. Annual state government spending on nutritional education was graciously provided by Kerry Anne McGeary; see McGeary (2009) for detailed information on this variable. We construct a series of time-varying dummy variables reflecting the extent of state clean indoor air laws, using the classification scheme of the 1989 Surgeon General's Report (US Department of Health and Human Services, 1989). The data come from a combination of the 1989 Surgeon General's Report and Impacteen. ${ }^{7}$ Annual state unemployment rates come from the Bureau of Labor Statistics.

After merging the individual- and state-level datasets and dropping observations with missing variables, our sample size is 2,428,009. Table 1 gives summary statistics for the cigarette price, BMI, and health behavior variables used in this study, while Table A1 in the online appendix reports the summary statistics for the control variables. Some of the health behavior-related variables are only available for a portion of the sample; Table 1 indicates this where relevant.

\section{Empirical Analysis}

\section{A. Baseline Model}

We study the effect of cigarette price on body mass index (BMI) by modifying the preferred specification of Courtemanche (2009) to account for heterogeneity throughout the BMI distribution and by race, sex, age, and education. We adopt the approach of Courtemanche (2009) because he showed that the other methodologies used in the literature all point to negative long-run average effects of cigarette costs on BMI and probability of being obese once lagged costs are included in the model. After considering a number of specifications, Courtemanche $(2009 ;$ p. 794) ultimately settled on a preferred model that included as covariates average state cigarette prices over years $t$ to $t-1, t-2$ to $t-3$, and $t-4$ to $t-5$; demographic controls; and state and year fixed effects. We adopt this approach as a starting point in our analysis of heterogeneity. Our only change is that, in order to enable clearer presentation of the quantile and subsample results, we use one six-year moving average spanning $t$ to $t-5$ years rather than three two-year averages; this does not meaningfully impact the results. The six-year moving average of cigarette price increased significantly over time, more than doubling between 1984 and 2005 ( $\$ 1.46$ versus $\$ 3.89$ per pack). Extensive price variation also exists between states. For example, the six-year average cigarette price in 2005 ranged from $\$ 3.18$ per pack in Kentucky to $\$ 5.20$ in New York.

We evaluate the heterogeneity in the cigarette price effect at various locations of the BMI distribution by unobserved characteristics that affect BMI using quantile regression (QR). The QR model estimates the conditional distribution of BMI quantiles as a function of cigarette price and other covariates and identifies the price effects on BMI at various quantiles. The effects of the explanatory variables on the conditional quantiles, which we refer to as quantile effects $(\mathrm{QE})$, may be interpreted as price effects at different levels of the net value of all unobserved characteristics that determine the rank of an individual on the BMI distribution conditional on the observed characteristics (Chernozhukov, V., Hansen,

\footnotetext{
${ }^{7}$ The data and classification scheme have been used in prior research, such as Eriksen and Chaloupka (2007). We thank Frank Chaloupka, Michael Eriksen, and Gary Giovina for directing us to them.
} 
2004; Wehby et al., 2009). Such unobserved characteristics may include biologic/genetic, demographic, socioeconomic, and environmental factors. Our QR model can be characterized as follows (Chernozhukov, V., Hansen, 2004):

$$
W_{i s t}=\mathrm{Q}\left(\bar{P}_{s t}, \mathbf{X}_{i s t}, \mathbf{S}_{s}, \boldsymbol{Y}_{t}, U_{i s t}\right),
$$

where $W_{i s t}$ is the BMI of individual $i$ living in state $s$ in year $t$, while $\mathrm{Q}$ is the conditional quantile distribution. $\bar{P}_{S t}$ is average inflation-adjusted state cigarette price, defined as

$$
\bar{P}_{s t}=\frac{P_{s t}+P_{s, t-1}+P_{s, t-2}+P_{s, t-3}+P_{s, t-4}+P_{s, t-5}}{6} .
$$

$\mathbf{X}_{i s t}$ is a vector of individual demographic and socioeconomic characteristics consisting of age (and age squared), race dummies (white, black, and other race), sex, a dummy for married, dummies for education (less than a high school degree, high school degree but no further, some college, and college graduate), and inflation-adjusted household income (and income squared). $\mathbf{S}_{s}$ and $\mathbf{Y}_{t}$ denote dummy variables for state of residence and survey year. Finally, $U_{i s t} \sim(0,1)$ is a uniformly distributed ranking variable that determines individual $i$ 's rank on the BMI distribution conditional on the control variables, and therefore represents the net level of all unobserved characteristics that are relevant for this rank. In other words, individuals with different values of $U$ but similar observable characteristics would have different ranks on the BMI distribution. For each quantile $q$ of BMI such that $0<q<1, \mathrm{Q}\left(\bar{P}_{s t}\right.$, $\left.\mathbf{X}_{i s t}, \mathbf{S}_{s}, \mathbf{Y}_{t}, q\right)$ is the value of the conditional $q$ th BMI quantile. Since the effects of cigarette prices on BMI are estimated holding $U$, the net level of the unobserved characteristics relevant to the individual's BMI rank, constant at $q$, the QE at different $q$ represent effects on BMI at different levels of these characteristics (Chernozhukov, V., Hansen, 2004). In other words, the heterogeneity in effects of cigarette prices and other model variables on BMI by $U$ can be evaluated by estimating the $\mathrm{QE}$ at different quantiles as:

$$
W_{i s t}=\mathrm{Q}\left(\alpha_{0 q}+\beta_{q} \bar{P}_{s t}+\mathbf{X}_{i s t} \boldsymbol{\gamma}_{q}+\mathbf{S}_{s} \boldsymbol{\lambda}_{q}+\boldsymbol{Y}_{t} \boldsymbol{\kappa}_{q}\right)
$$

where $\beta_{q}$ represents the effects of cigarette prices at quantile $q$ of BMI, $\boldsymbol{\gamma}_{q}$ is a vector of the effects of the control individual-level variables, and $\boldsymbol{\lambda}_{q}$ and $\boldsymbol{\kappa}_{q}$ are vectors of the state and year fixed effects.

The state and year fixed effects capture unobserved state-level determinants of BMI that are stable over time as well as unobserved year-to-yearchanges in BMI that are shared by all states. A natural concern with this model is whether changes over time in unobserved statelevel characteristics, such as demand for health, could lead to omitted variable bias by influencing both cigarette prices and population weight. Courtemanche (2009) performed a large number of robustness checks to help rule out this possibility in the context of mean effects. In Section IIIE, we will conduct our own series of robustness checks which show that the results are overall insensitive to different specifications.

QR is estimated by minimizing a weighted sum of the absolute deviations of the actual BMI values from conditional values for each $q$ across all individuals in the sample (Koenker and Bassett, 1978; Koenker and Hallock, 2001):

$$
\min \left[q \sum_{W_{i} \geq \mathrm{Q}_{i}}^{n}\left|W_{i}-\mathrm{Q}_{i}\right|+(1-q) \sum_{W_{i}<\mathrm{Q}_{i}}^{n}\left|W_{i}-\mathrm{Q}_{i}\right|\right]
$$

where $n$ is the total number of individuals. 
We estimate the $Q R$ model for the following five BMI quantiles that are expected to provide adequate coverage of the entire BMI distribution: $0.1,0.25,0.5,0.75$ and 0.9 . In order to test for differences in cigarette price effects between these quantiles in the main model, we estimate the variance-covariance matrices of the QR models simultaneously using 200 bootstrap replications (Hao and Naiman, 2007).

As a reference point, we also estimate the BMI function using OLS in order to compare the cigarette price effects at the mean of BMI as has been calculated in previous studies to the effects at the five quantiles. For OLS, we estimate standard errors that are heteroskedasticity-robust and clustered by state (Moulton, 1986). Sampling probability weights cannot be employed in the QR estimation. In unreported regressions, we estimate alternative OLS models that use the sampling probability weights and find similar cigarette price effects to the unweighted models. We estimate all models for the total sample and stratified by race, education level, age, and sex.

\section{B. Baseline Model Results}

The first row of Table 2 reports the marginal effect of cigarette price averaged over a sixyear period on the mean of BMI and the five BMI quantiles for the total sample. ${ }^{8}$ For the mean effect, we also show the elasticity (in brackets) to aid the reader's assessment of economic significance. ${ }^{9}$ Cigarette price has a significant negative effect on BMI that monotonically increases (in absolute value) by the quantile order and is significantly larger at higher than lower quantiles in the total sample and most stratified samples. In the total sample, a one-dollar increase in price reduces BMI by $0.12,0.2,0.28,0.37$ and 0.39 units at the $0.1,0.25,0.5,0.75$ and 0.9 quantiles, respectively; these effects are significantly different from each other (at $\mathrm{p}<0.01$ ). The effect at the mean is generally similar to the effect at the median ( 0.5 quantile). Since the health benefits from weight loss are greatest for overweight and obese individuals, these results imply that higher cigarette prices are even more beneficial for health than focusing on mean effects alone would suggest.

\section{Effects by Demographic and Socioeconomic Characteristics}

Table 2 also reports the cigarette price effects on BMI mean and quantiles for subsamples defined by race, sex, age, and education. Stratifying by race, the largest price effects are for black individuals, especially those at high BMI quantiles. This is consistent with previous studies finding stronger price effects on smoking behaviors among blacks (e.g. Farrelly et al 2001; Nonnemaker and Farrelly, 2011). A one-dollar increase in cigarette price reduces BMI by 0.76 and 0.58 units at the 0.9 and 0.75 quantiles, respectively, compared to a decrease of 0.17 units at the 0.1 quantile and 0.36 units at the median (with a 0.36 unit decrease at the mean). Smaller cigarette price effects are observed for whites and those of a race other than white or black. Among whites, a one-dollar price increase reduces BMI by 0.32, 0.28 and 0.14 at the $0.9,0.5$ and 0.1 quantiles. Among individuals of another race, a one dollar increase in price reduces the 0.9 and 0.5 quantiles of BMI by 0.34 and 0.17 units, but has a smaller and insignificant effect at the 0.1 quantile. Price effects are overall significantly different between the quantiles in the race-specific models.

Stratifying by highest school grade completed reveals that education increases price responsiveness at BMI quantiles 0.1 through 0.75. For example, cigarette prices have small

\footnotetext{
${ }_{9}^{8}$ Online Appendix Table A2 gives the marginal effects for the control variables.

${ }^{9}$ The elasticities for the quantile regressions are available upon request but we do not present them here because they can be misleading. Since the marginal effect is divided by a larger baseline BMI at higher than lower quantiles when calculating the elasticity, differences in elasticities between quantiles may not accurately reflect the differences in marginal effects. For instance, focusing on elasticities would treat a five pound weight gain by a 100 pound person as equivalent to a fifteen pound weight gain by a 300 pound person, even though the latter is likely of more consequence for health.
} 
and insignificant effects on BMI at the 0.1 quantile among individuals of high school education or less, but have a larger and significant effect at the 0.1 quantile for individuals of higher education with a one dollar increase in price reducing BMI by 0.13 units. Similarly, at the median cigarette prices have a small and insignificant impact on the BMI of individuals who did not complete high school, but have larger and significant effects for more educated individuals with a $0.23-0.26$ decrease in BMI. In contrast, cigarette prices have the largest impacts at the 0.9 quantile for individuals who have less than a high school education and for college graduates, decreasing their BMI by 0.44- 0.45 units per one-dollar price increase, compared to decreases of 0.2-0.3 units for individuals with intermediate education. The cigarette price effects are significantly different between the five quantiles for all education subgroups.

The finding that the BMI response to cigarette prices generally strengthens with education may seem contradictory to prior research finding a larger cigarette price elasticity of smoking for less educated than more educated individuals (e.g. Chaloupka, 1991). However, our results are consistent with the theory that more educated individuals are more efficient and motivated in capitalizing on an improvement in one health behavior such as reducing or avoiding smoking to adopt a healthier lifestyle in general and improve other health behaviors such as exercise. In other words, cigarette prices could have the strongest effect on smoking for those with low education levels, but smoking changes could have the strongest effect on BMI for those with high education levels.

Cigarette prices have larger effects for younger ( 59 ) than older ( 200 ) adults at the mean and all evaluated quantiles. This is consistent with previous studies showing lower cigarette price elasticities of smoking for older adults (e.g. Chaloupka, 1991; Liu, 2010). The largest cigarette price effects on BMI are at the 0.9 quantile for middle age adults (40-59), where a one-dollar price increase reduces BMI by 0.82 units, compared to a 0.6-unit BMI decrease for younger adults $(<40)$ and a much smaller and insignificant decrease for older adults. The price effects at the mean are also largest for middle age adults, where BMI is reduced by 0.45 units with a one-dollar price increase, compared to a 0.36-unit decrease for individuals younger than 40 years. Except for a small effect at the 0.25 quantile, cigarette prices are generally not statistically associated with BMI for older adults. The price effects are significantly different between the five quantiles for young and middle age adults.

Finally, cigarette prices have slightly larger effects for females than males, with the largest difference at the BMI median, where a one-dollar price increase reduces BMI by 0.33 units for females and 0.23 units for males. This is consistent with prior findings of a larger cigarette price elasticity of smoking among females (Farrelly et al., 2001; Tauras and Chaloupka, 1999). Similarly to the total sample and most of the other stratified samples, our estimated cigarette price effects on BMI are overall significantly larger at higher than lower quantiles for both females and males.

\section{Implications for Disparities}

There are significant disparities in obesity and severe obesity by race, education, age, and sex (see Table A3 in the online appendix). About $30.4 \%$ of black individuals are obese (including $12 \%$ who are severely obese) compared to $18.3 \%$ of whites $(5.7 \%$ severely obese). Obesity and severe obesity rates decrease with education, with $24.7 \%$ of individuals with less than a completed high school education being obese (8.6\% are severely obese) compared to $15.2 \%$ of college graduates ( $4.4 \%$ severely obese). Obesity and severe obesity rates are highest for middle age adults (40-59) at $23.4 \%$ and $8.1 \%$, and lowest for young adults $(<40)$ at $15.7 \%$ and $5.2 \%$. Finally, severe obesity rates are higher among women than men $(7.2 \%$ versus $5.1 \%)$. 
Given the heterogeneity in the cigarette price effect observed across these groups, a natural question is whether rising cigarette prices have ameliorated or worsened these disparities. We attempt to quantify the impacts on disparities in Table 3. For each group, we estimate a linear probability model for obesity using the same main specification described above for the BMI function. From that regression, we predict the obesity rate in 2005 (the last year of our sample) at both 1984 (the first year of our sample) cigarette prices and 2005 cigarette prices (prices are still averages over the current and past five years). We then compute the predicted obesity rate disparity between each group and the group with the lowest obesity rate (e.g. blacks or other race versus whites) at both 2005 and 1984 prices. Finally, we calculate the difference (change) in these predicted obesity rate disparities. These difference estimates quantify the degree to which obesity disparities are larger or smaller than they would have been if cigarette prices had stayed at 1984 levels. We obtain standard errors for the predicted disparities and their changes due to cigarette prices using 200 bootstrap replications. We then repeat the same analysis for severe obesity.

Several statistically significant results emerge. The increase in cigarette price between 1984 and 2005 has decreased the disparity in the severe obesity rate between blacks and whites by about 4.8 percentage points, a striking $36 \%$ of the baseline disparity. In contrast, rising cigarette prices have increased the obesity rate disparity between college graduates and individuals with less than a high school degree by about 3.8 percentage points. Furthermore, changes in cigarette prices decreased the disparity in obesity and severe obesity rates between middle age and young adults by about 1.7 and 1.3 percentage points, respectively. However, cigarette price changes have increased the disparity in obesity and severe obesity rates between older ( 260 ) and young adults by about 4.6 and 2.4 percentage points. Finally, cigarette price changes reduced the disparity in severe obesity rates between females and males.

\section{E. Robustness Checks}

This subsection performs several robustness checks to evaluate the sensitivity of the results to different sets of control variables and cigarette cost measures. The first adds several timevarying state-level controls: fitness centers per capita, nutrition education spending, clean indoor air laws, and unemployment rate. ${ }^{10}$ This specification aims to control for the most obvious potential sources of omitted variable bias. Fitness centers proxy for unobservable population attitudes toward healthy lifestyles and demand/supply for exercise facilities, nutrition education spending proxies for state obesity-control policies, clean indoor air laws proxy for other state tobacco-control policies besides cigarette taxes, and unemployment rate proxies for state economic conditions.

The second robustness check aims to control for time-varying state unobservable characteristics more generally by including linear state-specific time trends.

Multicollinearity is a problem with this model: controlling for state trends along with state and year fixed effects and the control variables results in a variance inflation factor of 133 , well above the level of 10 at which the level of multicollinearity is considered problematic (Wooldridge, 2006: 203-204). ${ }^{11}$ Accordingly, the resulting estimates are too imprecise to be useful. As a solution, in the regressions with state-specific time trends we replace the year fixed effects with a quadratic time trend, an approach that has been taken by other papers in the obesity literature facing the same problem (Chou et al., 2002; Courtemanche, 2011). ${ }^{12}$

\footnotetext{
${ }^{10}$ Following McGeary (2009), we do not scale nutrition education spending by population, but we have verified that this does not impact our conclusions.

${ }^{11}$ Put differently, the state and year fixed effects, state trends, and control variables together explain over $99 \%$ of the variation in cigarette price.
} 
The third robustness check includes more detailed individual-level controls. Specifically, we add a set of dummies for number of adults in the household (one for each number through 4 , with 5 or more as the reference category), and also control for age and income more flexibly. For age, we include dummies for each five year increment from 25 until 85 years, then another dummy for over 85 (18 to 25 is the reference category). We model income with dummies for each $\$ 5,000$ increment up to $\$ 100,000$, and another dummy for income over $\$ 100,000$ (income $\leq \$ 5000$ as the reference category).

The fourth robustness check uses an 8-year rather than 6-year moving average for cigarette price (unreported regressions also tried 10- and 12-year moving averages, obtaining similar results). Finally, we return to the six-year moving average but use per-pack state cigarette excise tax rates instead of prices.

Table 4 summarizes the sensitivity of our key results to these alternative specifications, while online Appendix Tables A4 to A8 report the full array of cigarette price (or tax) effects. The key conclusions from the baseline model generally hold up well. The only exception is that in the model with state trends the largest effect (both at the mean and several quantiles) by age is for those over 60 rather than for those ages 40 to 59 . However, recall that the state trends model replaces the year dummies with a quadratic time trend. If we add a cubic term to the time trend, the age stratifications once again lead to the same conclusion as the baseline model: the strongest effect is for the 40 to 59 group, with essentially no response among those 60 and over.

\section{F. Cigarette Price Effects on Smoking and BMI-Related Behaviors Across the BMI Distribution}

Conceptually, the reduced-form impact of cigarette price on weight is the product of a complicated chain of events: 1) the effect of price on smoking, which could occur along either the extensive or intensive margins; ${ }^{13}$ 2) the effect of smoking on behaviors related to calorie intake or expenditure; and 3) the effects of these behaviors on weight. Heterogeneous impacts across BMI levels and demographic and socioeconomic characteristics are possible at all three of these stages. This section therefore investigates how the effects of cigarette prices on variables related to these mechanisms vary with BMI in an attempt to shed some light on the clearest source of heterogeneity observed in the preceding sections: the strengthening of the cigarette price effect on BMI moving from left to right across the BMI distribution. We do this by stratifying the sample into BMI quintiles and estimating for each quintile the impact of the six-year moving average of cigarette price on the BMI-related health behavior variables from Table $1 .^{14}$

The results from this subsection should be interpreted subject to several caveats: 1) several of the dependent variables are available for only a portion of the sample, as shown in Table 1 , so the estimates are relatively imprecise; 2 ) stratifying by BMI means stratifying by an

\footnotetext{
${ }^{12}$ In unreported regressions we verified that our baseline results are generally not sensitive to replacing the year dummies with a quadratic time trend, suggesting that such a switch is not inappropriate here.

${ }^{13}$ Given the pooled cross-sectional nature of the BRFSS, we are only able to examine respondents' smoking at one point in time as opposed to actually observing initiation and cessation. However, prior research suggests that cigarette prices do influence both initiation and cessation. For instance, Nonnemaker and Farrelly (2011) report a consistent effect of cigarette prices on reducing youth smoking initiation, with larger effects among blacks. Liu (2010) finds significant cigarette price effects on initiation up to age 44. That study also finds a positive and significant effect of cigarette prices on quitting smoking, especially among individuals age 45 and older. DeCicca et al. (2008) report increased smoking cessation with higher taxes among young adults. Tauras and Chalupka (1999) also find that higher cigarette prices increase smoking cessation among young adults (for both men and women).

${ }^{14} \mathrm{We}$ choose quintiles because each subsample therefore contains one of the locations on the BMI distribution evaluated by our earlier quantile regressions (the first quintile contains the $10^{\text {th }}$ percentile, the second contains the $25^{\text {th }}$ percentile, the third contains the
$50^{\text {th }}$ percentile, the fourth contains the $75^{\text {th }}$ percentile, and the fifth contains the $90^{\text {th }}$ percentile). Unreported analyses further stratify by race, education, age, and gender but this generally results in sample sizes too small to obtain meaningful estimates.
} 
endogenous variable; 3 ) the subsample approach is not an exact parallel of the quantile regression approach used for BMI; and 4) we do not have complete food diaries and therefore rely on proxies for diet healthfulness. For these reasons, further research is needed to fully understand the mechanisms behind the negative long-run effect of cigarette price on body weight and its heterogeneity. Nonetheless, some interesting preliminary results emerge.

We begin by estimating the impact of price on three smoking variables. The first, a dummy for whether the individual currently smokes, relates to smoking participation. Next, number of cigarettes smoked per day conditional on being a current smoker captures intensity. ${ }^{15}$ Finally, we estimate an "overall" effect of price on smoking - encompassing both the extensive and intensive margins - by using cigarettes per day among the whole sample, with zero values assigned to non-smokers. ${ }^{16}$

The first three columns of Table 5 report the results for these smoking variables. Higher sixyear average cigarette prices lead to statistically significant reductions in all three smoking variables for the full sample and all BMI quintile groups. Cigarette price has the strongest impact on the probability of currently smoking for the lowest BMI quintile. In contrast, the effect on cigarettes smoked per day among smokers generally rises with BMI and is strongest in the highest quintile. Interestingly, the way in which the cigarette price effect on BMI changes across the BMI distribution more closely mirrors the pattern for the intensive margin of smoking than the pattern for the extensive margin. ${ }^{17}$ The overall effect of cigarette price effect on cigarettes smoked per day for the whole sample - shown in the third column - exhibits an inverted U-shaped pattern in relationship to BMI quintile with negative impacts that are strongest at the two tails of the BMI distribution.

The last four columns of Table 5 show the results for the variables related to exercise, eating, and drinking. A $\$ 1$ increase in the six-year moving average of cigarette price leads to a marginally significant increase in exercise for the whole sample, with a sizeable magnitude that represents $12 \%$ of the sample mean exercise level. Importantly, the cigarette price effect on exercise across the BMI distribution exhibits the same inverted U-shaped pattern of heterogeneity observed for overall smoking (cigarettes per day for the whole sample). In contrast, there is little evidence that any of the variables related to calorie consumption provide viable mechanisms. Cigarette price does have a marginally significant influence on fruit and vegetable consumption for the full sample, but the effect is relatively small (2.7\% increase of the sample mean with $\$ 1$ price increase) and the pattern of heterogeneity does not match those in price effects on either smoking or BMI. There is no evidence that cigarette price influences calorie/fat restriction or alcohol intake, either for the full sample or any subsamples.

It is noteworthy that the pattern of heterogeneity observed in the cigarette price effect on exercise (strong effects at both tails) differs from the pattern observed in the cigarette price effect on BMI (strongest effect at the right tail). This suggests that heterogeneity in price effects on behaviors cannot fully account for the results, and that heterogeneous effects of behaviors on BMI play a role as well. For instance, if individuals who are already thin lose

\footnotetext{
15 Whether the individual is a smoker is available in all survey years while cigarettes smoked per day is only available through 2000. For consistency, we only use the years up to 2000 in all the smoking regressions.

${ }^{16}$ We estimate linear models for all the regressions in this section, but have verified that the results are similar using probit models for the binary dependent variables and Poisson regression for the counts.

${ }^{17}$ Online Appendix Table A9 takes a different approach to assessing whether changes in smoking along the intensive margin play a role in explaining the cigarette price effect on weight: restricting the sample to current smokers and estimating the baseline quantile regression model. The evidence suggests that cigarette price influences the BMI of smokers for some subsamples, especially at higher quantiles (quantile 0.75 ), consistent with smoking intensity contributing to our results.
} 
less weight from a specified increase in exercise than those who start with substantial excess body fat, it would make sense that the similarly-sized cigarette price effects on exercise at the two tails of the BMI distribution lead to greater weight loss at the right tail. Indeed, if we run a quantile regression of BMI on minutes of exercise and the individual-level control variables, the association between exercise and BMI steadily becomes more negative across the BMI distribution. ${ }^{18}$

\section{Discussion}

Whether rising cigarette costs have had the unintended consequence of contributing to the rise in obesity has become the subject of considerable debate, with recent research showing that the average effect of cigarette prices on BMI is surprisingly negative using all methodologies from the literature if the effect is allowed to occur gradually over six or more years (Courtemanche, 2009). In this study, we build on Courtemanche (2009) by showing that the relationship between cigarette prices and BMI becomes more strongly negative with body weight and varies across subgroups defined by race, education, age, and sex,. We also assess the implications for disparities and conduct a preliminary investigation of the behavioral mechanisms that might contribute to this heterogeneity.

The finding that individuals at higher BMI quantiles are significantly more responsive to cigarette prices than those at lower BMI quantiles has important implications. Since weightrelated illnesses and their associated medical expenditures are concentrated amongst the obese and severely obese, the health benefits and fiscal savings from higher cigarette taxes are greater than one would infer from previous estimates of effects at BMI. ${ }^{19}$ The extent of the heterogeneity is non-trivial. To illustrate, for the full sample the mean effects underestimate the effects at the 0.9 percentile by about $28 \%$ and overestimate the effects at the 0.1 percentile by more than $130 \%$. Even larger differences between mean and quantile effects are observed in certain groups: for example, mean effects underestimate the effect at the 0.9 quantile for blacks by about $50 \%$.

Additionally, our study is the first to evaluate the implications of cigarette price increases over the past decades for obesity disparities across major demographic and socioeconomic characteristics. Perhaps most notably, we find the greatest weight response to cigarette prices among blacks, which has translated into a statistically and economically significant reduction in the severe obesity gap between blacks and whites. This result, combined with prior research finding stronger price effects on smoking behaviors among blacks (e.g. Farrelly et al 2001; Nonnemaker and Farrelly, 2011), suggests that cigarette taxation could play an important role in reducing racial disparities in health in the future - both directly through smoking and indirectly through BMI. Beyond race, our results also suggest that the rise in cigarette prices has mitigated weight-related disparities by gender, had mixed effects on disparities by age, and increased disparities by education.

A more conservative interpretation of the policy implications of our results would simply be that they provide the most complete evidence to date that policymakers do not need to fear the unintended consequence of weight gain when considering cigarette taxes. Prior research has only been able to reach this conclusion with regard to population averages; even if cigarette prices do not increase population weight on average they still might for certain vulnerable populations, such as those with self-control problems or a genetic predisposition

\footnotetext{
${ }^{18}$ The regression coefficients for minutes of exercise per day are $-0.00005,-0.001,-0.003,-0.006$, and -0.009 at the $0.1,0.25,0.5$, 0.75 , and $0.9 \mathrm{BMI}$ quantiles, respectively. We have no appropriate instruments for exercise to formally estimate its causal effects of BMI, so these associations should be taken as preliminary.

${ }^{19}$ Cawley and Meyerhoefer (2010) note that the obese and severely obese are responsible for the largest share of medical expenditures.
} 
to be overweight. Our results help to rule out this possibility. Between all the subsamples and quantiles, Table 2 provides estimates for "78 categories" of individuals, and cigarette prices do not significantly increase BMI for a single one of them. The concurrent trends in smoking and obesity therefore appear to have been driven by coincidental population-level changes rather than causal effects of smoking reductions on increasing body weight. ${ }^{20}$ The decrease in smoking rates is thought to have been primarily driven by a combination of increased knowledge of the health consequences of smoking and higher cigarette prices. The rise in obesity may have been the result of unrelated factors such as an increasingly sedentary lifestyle, greater fast-food and restaurant availability, food advertisements, improvements in food production technology, falling real food prices, and historically cheap gasoline. $^{21}$

Another contribution of our study is to provide some insight into the mechanisms through which the negative effect of cigarette prices on BMI occurs and why it is heterogeneous. First, the effect appears to reflect more than simply the consequences of quitting smoking the intensive margin appears to play a role as well. The pattern of how the cigarette price effect on BMI varies across the BMI distribution is more similar to the pattern for smoking intensity than that for smoking participation. Moreover, the similarity of the patterns for overall smoking and exercise would not have emerged without accounting for smoking intensity when creating our overall smoking variable. Our results also provide the strongest evidence to date that exercise is one pathway through which the surprising long-run negative effect of cigarette prices on weight occurs. Reducing or quitting smoking could lead to more exercise by increasing lung capacity, enthusiasm about health, leisure time, or expected longevity. Over a long enough time horizon, the additional exercise appears to counteract the biological effects on appetite and metabolism, leading to net weight loss, particularly among individuals at the higher weight quantiles.

Finally, our study has implications for the broader literature on the economic causes of obesity. If cigarette prices have heterogeneous effects on BMI both across BMI levels and demographic and socioeconomic characteristics, then it stands to reason that the influences of other environmental characteristics may be heterogeneous as well. Interesting insights may emerge from applying a similar methodological approach to the one used here to study heterogeneity in the effects of food prices, restaurant density, gasoline prices, and other economic factors on BMI.

\section{Acknowledgments}

Data analysis was supported in part by NIH/NIDCR grant R01 DE020895.

\section{References}

Anderson ML, Matsa DA. Are Restaurants Really Supersizing America? American Economic Journal: Applied Economics. 2011; 3(1):152-188. doi: http://www.aeaweb.org/aej-applied/.

Andreyeva Y, Kelly I, Harris J. Exposure to food advertising on television: Associations with children's fast food and soft drink consumption and obesity. Economics \& Human Biology. 2011; 9(11):12.

\footnotetext{
${ }^{20}$ Alternatively, the rise in obesity could have causally reduced smoking rates due to increasing concerns about health problems resulting from obesity such as hypertension and heart disease. While research suggests that obesity may increase smoking initiation among female adolescents (Cawley et al., 2004; Rees and Sabia, 2010), the causal effect of weight on smoking among adults remains unexplored.

${ }^{21}$ For a sample of research exploring these possibilities, see Lakdawalla and Philipson (2002), Philipson and Posner (2003), Cutler et al. (2003), Rashad et al, (2006), Eid et al. (2008), Chou et al. (2008), Currie et al. (2010), Dunn (2010), Zhao and Kaestner (2010), Courtemanche and Carden (2011), Anderson and Matsa (2011), Andreyeva et al. (2011), Courtemanche (2011), and Goldman et al. (2011)
} 
Baum CL. The effects of cigarette costs on BMI and obesity. Health Economics. 2009; 18(1):319.10.1002/hec.1340 [PubMed: 18241088]

Cawley, J. PhD Dissertation. University of Chicago; 1999. Rational addiction, the consumption of calories, and body weight.

Chicago IL, Cawley J, Markowitz S, Tauras J. Lighting up and slimming down: The efects of body weight and cigarette prices on adolescent smoking initiation. Journal of Health Economics. 2004; 23(2):293-311. [PubMed: 15019756]

Cawley, J.; Meyerhoefer, C. National Bureau of Economic Research Working Paper, No 16467. 2010. The medical care costs of obesity: An instrumental variables approach.

CDC. Health, United States, 2009: With Special Feature on Medical Technology. National Center for Health Statistics; 2010.

Chaloupka F. Rational Addictive Behavior and Cigarette-Smoking. Journal of Political Economy. 1991; 99(4):722-742.

Chaloupka, FJ.; Grossman, M. National Bureau of Economic Research Working Paper Series, No 5740. 1996. Price, Tobacco Control Policies and Youth Smoking.

Chen H, Hansen MJ, Jones JE, Vlahos R, Anderson GP, Morris MJ. Long-term cigarette smoke exposure increases uncoupling protein expression but reduces energy intake. Brain Res. 2008; 1228:81-88. S0006-8993(08)01537-0 [pii]. 10.1016/j.brainres.2008.06.067 [PubMed: 18619427]

Chen H, Vlahos R, Bozinovski S, Jones J, Anderson GP, Morris MJ. Effect of short-term cigarette smoke exposure on body weight, appetite and brain neuropeptide $\mathrm{Y}$ in mice. Neuropsychopharmacology. 2005; 30(4):713-719. 1300597 [pii]. 10.1038/sj.npp.1300597 [PubMed: 15508020]

Chernozhukov V, Hansen. The effects of 401(k) participation on the wealth distribution: an instrumental quantile regression analysis. Review of Economics and Statistics. 2004; 86(3):735751.

Chou, S.; Grossman, M.; Saffer, H. National Bureau of Economic Research Working Paper, No 16467. 2002. An economic analysis of adult obesity: An instrumental variables approach.

Chou SY, Grossman M, Saffer H. An economic analysis of adult obesity: results from the Behavioral Risk Factor Surveillance System. Journal of Health Economics. 2004; 23(3):565-587. S0167-6296(04)00020-7 [pii]. 10.1016/j.jhealeco.2003.10.003 [PubMed: 15120471]

Courtemanche $\mathrm{C}$. Rising cigarette prices and rising obesity: coincidence or unintended consequence? Journal of Health Economics. 2009; 28(4):781-798. S0167-6296(09)00037-X [pii]. 10.1016/ j.jhealeco.2009.03.003 [PubMed: 19356816]

Courtemanche C. A Silver Lining? The Connection between Gasoline Prices and Obesity. Economic Inquiry. 2011; 49(3):935-957.10.1111/j.1465-7295.2009.00266.x [PubMed: 22022734]

Courtemanche C, Carden A. Supersizing supercenters? The impact of Walmart Supercenters on body mass index and obesity. Journal of Urban Economics. 2011; 69(2):165-181.10.1016/j.jue. 2010.09.005

Currie J, Della Vigna S, Moretti E, Pathania V. The Effect of Fast Food Restaurants on Obesity and Weight Gain. American Economic Journal: Economic Policy. 2010; 2(3):32-63. doi: http:// www.aeaweb.org/aej-policy/.

Cutler DM, Glaeser EL, Shapiro JM. Why have Americans become more obese? Journal of Economic Perspectives. 2003; 17(3):93-118.

DeCicca P, Kenkel D, Mathios A. Cigarette taxes and the transition from youth to adult smoking: Smoking initiation, cessation, and participation. Journal of Health Economics. 2008; 27(4):904917.10.1016/j.jhealeco.2008.02.008 [PubMed: 18513811]

Dina C, Meyre D, Gallina S, Durand E, Korner A, Jacobson P, Froguel P. Variation in FTO contributes to childhood obesity and severe adult obesity. Nat Genet. 2007; 39(6):724-726. [PubMed: 17496892]

Dunn RA. The Effect of Fast-Food Availability on Obesity: An Analysis by Gender, Race, and Residential Location. American Journal of Agricultural Economics. 2010; 92(4):1149_ 1164.10.1093/Ajae/Aaq041

Eid J, Overman HG, Puga D, Turner MA. Fat city: Questioning the relationship between urban sprawl and obesity. Journal of Urban Economics. 2008; 63(2):385-404.10.1016/j.jue.2007.12.002 
Eriksen M, Chaloupka F. The economic impact of clean indoor air laws. [Research Support, Non-U.S. Gov’t Review]. CA Cancer J Clin. 2007; 57(6):367-378.10.3322/CA.57.6.367 [PubMed: 17989131]

Farrelly MC, Bray JW, Pechacek T, Woollery T. Response by adults to increases in cigarette prices by sociodemographic characteristics. Southern Economic Journal. 2001; 68(1):156-165.

Flegal KM, Carroll MD, Ogden CL, Curtin LR. Prevalence and trends in obesity among US adults, 1999-2008. Jama. 2010; 303(3):235-241. 2009.2014 [pii]. 10.1001/jama.2009.2014 [PubMed: 20071471]

Frayling TM, Timpson NJ, Weedon MN, Zeggini E, Freathy RM, Lindgren CM, McCarthy MI. A common variant in the FTO gene is associated with body mass index and predisposes to childhood and adult obesity. Science. 2007; 316(5826):889-894. [PubMed: 17434869]

Goldman, D.; Lakdawalla, D.; Zheng, Y. Food prices and the dynamics of body weight. In: Grossman, M.; Mocan, N., editors. Economic Aspects of Obesity. Chicago, IL: University of Chicago Press; 2011.

Gruber J, Frakes M. Does falling smoking lead to rising obesity? Journal of Health Economics. 2006; 25(2):183-197. discussion 389-193. S0167-6296(05)00060-3 [pii]. 10.1016/j.jhealeco. 2005.07.005 [PubMed: 16165234]

Hao, L.; Naiman, D. Quantile Regression. Sage Publications; 2007.

Hedenstrom H, Malmberg P, Fridriksson HV. Reference values for lung function tests in men: regression equations with smoking variables. Ups J Med Sci. 1986; 91(3):299-310. [PubMed: 3811032]

Hjelmborg JB, Fagnani C, Silventoinen K, McGue M, Korkeila M, Christensen K, Kaprio J. Genetic influences on growth traits of BMI: a longitudinal study of adult twins. Obesity (Silver Spring). 2008; 16(4):847-852. [PubMed: 18239571]

Koenker R, Bassett G Jr. Regression Quantiles. Econometrica. 1978; 46(1):33-50.

Koenker R, Hallock KF. Quantile Regression. Journal of Economic Perspectives. 2001; 15(4):143156.

Kring SI, Holst C, Zimmermann E, Jess T, Berentzen T, Toubro S, Sorensen TI. FTO gene associated fatness in relation to body fat distribution and metabolic traits throughout a broad range of fatness. PLoS ONE. 2008; 3(8):e2958. [PubMed: 18698412]

Lakdawalla, D.; Philipson, T. National Bureau of Economic Research Working Paper No 8965. 2002. The Growth of Obesity and Technological Change: A Theoretical and Empirical Investigation.

Liu F. Cutting through the smoke: separating the effect of price on smoking initiation, relapse and cessation. Applied Economics. 2010; 42(23):2921-2939. Pii 908134774. 10.1080/00036840801964880

Loos RJ, Lindgren CM, Li S, Wheeler E, Zhao JH, Prokopenko I, Mohlke KL. Common variants near MC4R are associated with fat mass, weight and risk of obesity. Nat Genet. 2008; 40(6):768-775. [PubMed: 18454148]

McGeary, KA. National Bureau of Economic Research Working Paper Series, No 15001. 2009. The Impact of State-Level Nutrition-Education Program Funding on BMI: Evidence from the Behavioral Risk Factor Surveillance System.

Moulton BR. Random Group Effects and the Precision of Regression Estimates. Journal of Econometrics. 1986; 32(3):385-397.

Nonnemaker J, Finkelstein E, Engelen M, Hoerger T, Farrelly M. Have efforts to reduce smoking really contributed to the obesity epidemic. Economic Inquiry. 2009; 47:366-376.

Nonnemaker JM, Farrelly MC. Smoking initiation among youth: The role of cigarette excise taxes and prices by race/ethnicity and gender. Journal of Health Economics. 2011; 30(3):560-567.10.1016/ j.jhealeco.2011.03.002 [PubMed: 21477875]

Orzechowski, W.; Walker, R. The Tax Burden on Tobacco, Historical Compilation. Vol. 41. Arlington, Virginia: 2006.

Philipson TJ, Posner RA. The long-run growth in obesity as a function of technological change. Perspectives in Biology and Medicine. 2003; 46(3):S87-S107. [PubMed: 14563077]

Pinkowish M. Hand in glove: Smoking cessation and weight gain. Patient Care. 1999; 33:134. 
Qi L, Kraft P, Hunter DJ, Hu FB. The common obesity variant near MC4R gene is associated with higher intakes of total energy and dietary fat, weight change and diabetes risk in women. Hum Mol Genet. 2008; 17(22):3502-3508.10.1093/hmg/ddn242 [PubMed: 18697794]

Rashad I, Chou S, Grossman M. The super size of America: An economic estimation of body mass index and obesity in adults. Eastern Economic Journal. 2006; 32:133-148.

Rees DI, Sabia JJ. Body weight and smoking initiation: Evidence from Add Health. Journal of Health Economics. 2010; 29(5):774-777. [PubMed: 20708283]

Scuteri A, Sanna S, Chen WM, Uda M, Albai G, Strait J, Abecasis GR. Genome-wide association scan shows genetic variants in the FTO gene are associated with obesity-related traits. PLoS Genet. 2007; 3(7):e115. [PubMed: 17658951]

Sturm R. The effects of obesity, smoking, and drinking on medical problems and costs. Health Aff (Millwood). 2002; 21(2):245-253. [PubMed: 11900166]

Tauras, JA.; Chaloupka, FJ. National Bureau of Economic Research Working Paper Series, No 7262. 1999. Determinants of Smoking Cessation: An Analysis of Young Adult Men and Women.

US Department of Health and Human Services. A Report of the Surgeon General. U.S. Department of Health and Human Services, Public Health Service, Centers for Disease Control, Center for Chronic Disease Prevention and Health Promotion, Office on Smoking and Health; 1989. Reducing the Health Cunsequences of Smoking: 25 Years of Progress. DHHS Publication No. (CDC) 89-8411

Villalobos-Comparan M, Teresa Flores-Dorantes M, Teresa Villarreal-Molina M, Rodriguez-Cruz M, Garcia-Ulloa AC, Robles L, Canizales-Quinteros S. The FTO Gene Is Associated With Adulthood Obesity in the Mexican Population. Obesity. 2008; 16(10):2296-2301. [PubMed: 18719664]

Watson NF, Goldberg J, Arguelles L, Buchwald D. Genetic and environmental influences on insomnia, daytime sleepiness, and obesity in twins. Sleep. 2006; 29(5):645-649. [PubMed: 16774154]

Wehby GL, Murray JC, Castilla EE, Lopez-Camelo JS, Ohsfeldt RL. Quantile effects of prenatal care utilization on birth weight in Argentina. Health Economics. 2009; 18:1307-21. [PubMed: 19142894]

Willer CJ, Speliotes EK, Loos RJ, Li S, Lindgren CM, Heid IM, Hirschhorn JN. Six new loci associated with body mass index highlight a neuronal influence on body weight regulation. Nat Genet. 2009; 41(1):25-34. [PubMed: 19079261]

Wooldridge, J. Introductory Econometrics: A Modern Approach. South-Western College Pub; 2006.

Zhao ZX, Kaestner R. Effects of urban sprawl on obesity. Journal of Health Economics. 2010; 29(6): 779-787.10.1016/j.jhealeco.2010.07.006 [PubMed: 20832131] 
Table 1

Distribution of Key Variables

\begin{tabular}{lccc}
\hline Variable & Years & Sample Size & Mean (Std. Dev.) \\
\hline State average cigarette price over years t to t-5 & All & $2,428,009$ & $26.28(5.32)$ \\
State average cigarette tax over years t to t-5 & All & $2,428,009$ & $0.42(0.28)$ \\
Body mass index (BMI) & All & $2,428,009$ & $26.28(5.32)$ \\
Obese (BMI $\geq 30)$ & All & $2,428,009$ & $0.20(0.40)$ \\
Severely obese (BMI $\geq 35$ ) & All & $2,428,009$ & $0.06(0.24)$ \\
Currently smokes & $1984-2000$ & $1,316,034$ & $0.22(0.41)$ \\
Cigarettes smoked per day (current smokers only) & $1984-2000$ & 288,493 & $18.83(10.65)$ \\
Cigarettes smoked per day (whole sample; 0 for non- smokers) & $1984-2000$ & $1,312,995$ & $4.14(9.26)$ \\
Minutes of exercise per day & $1984-2000$ & 928,060 & $26.39(39.38)$ \\
Fruits and vegetables per day & $1990-2003$ & $1,050,985$ & $3.73(1.83)$ \\
Indicator for restricting calories/fat to lose or maintain weight & $1985-2003$ & $1,162,221$ & $0.48(0.50)$ \\
Alcoholic drinks per month & All & $2,106,547$ & $10.28(23.04)$ \\
\hline
\end{tabular}




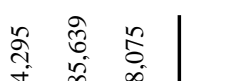

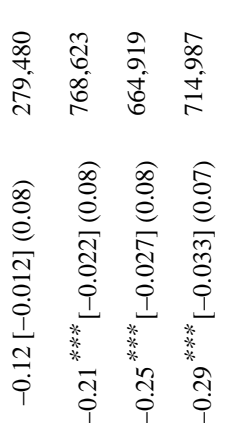

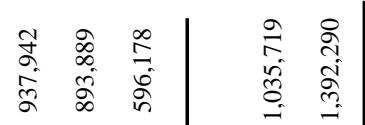

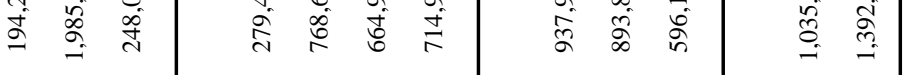

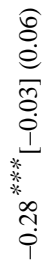

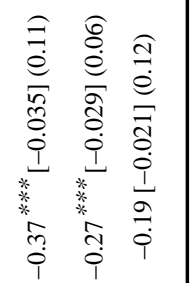

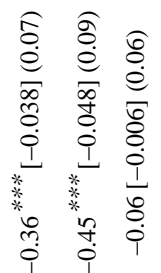

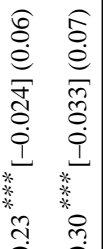

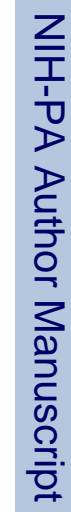

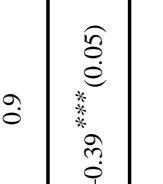

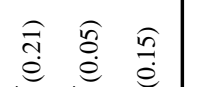

$\stackrel{6}{\circ} \stackrel{0}{\circ} \subseteq \stackrel{0}{\circ}$

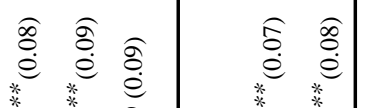

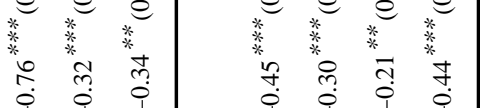

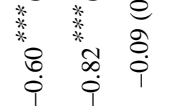

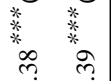

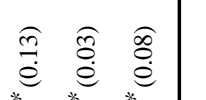

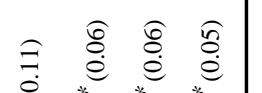

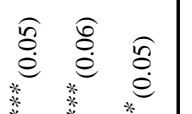

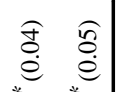

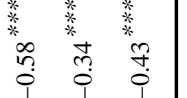

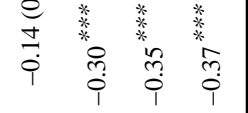

$\begin{array}{ccc}* & * & * \\ * & * & * \\ n & n & 0 \\ n & 0 & 0 \\ i & i & 1\end{array}$

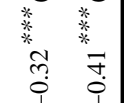

ป气

ôे

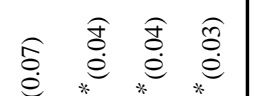

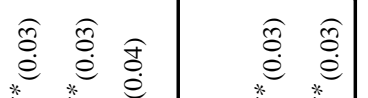

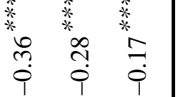

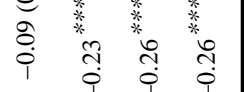

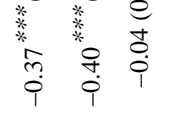

*ึ)

हิ

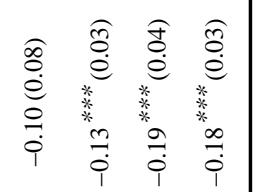

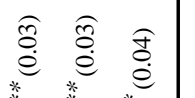

ڤิ)

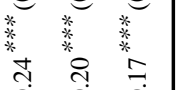

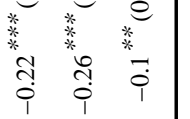

类

高 衤

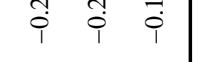

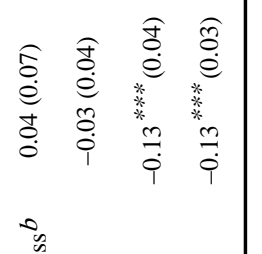

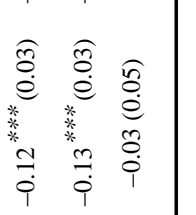

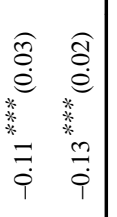

颃

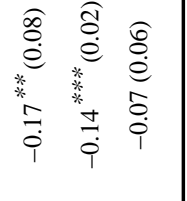

들. 


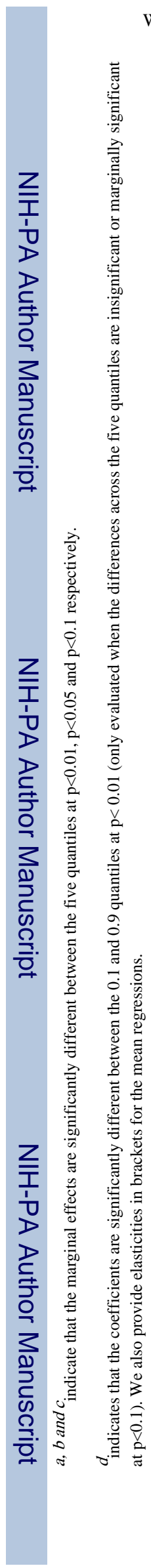

Wehby and Courtemanche

Page 19 D. 


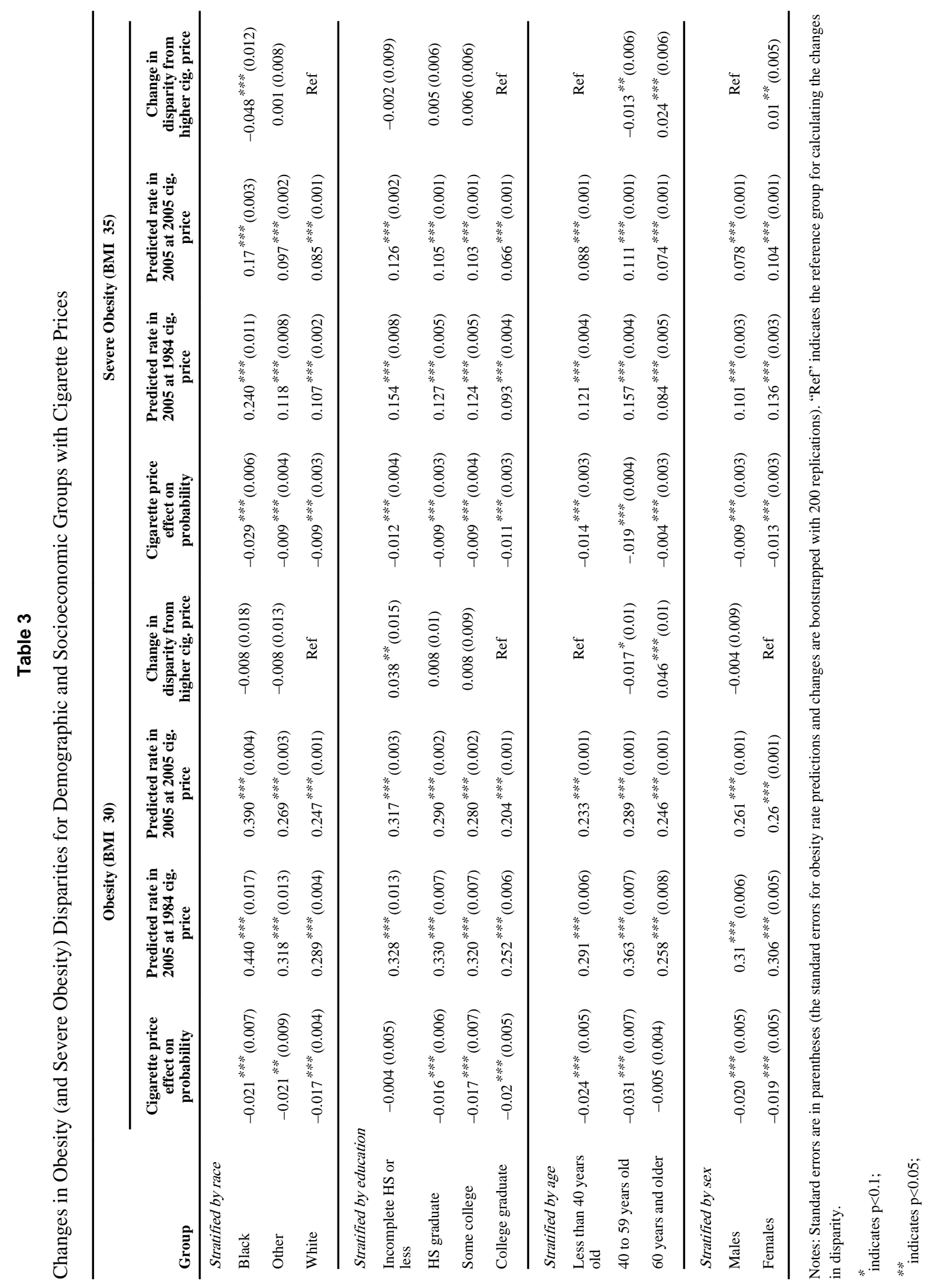




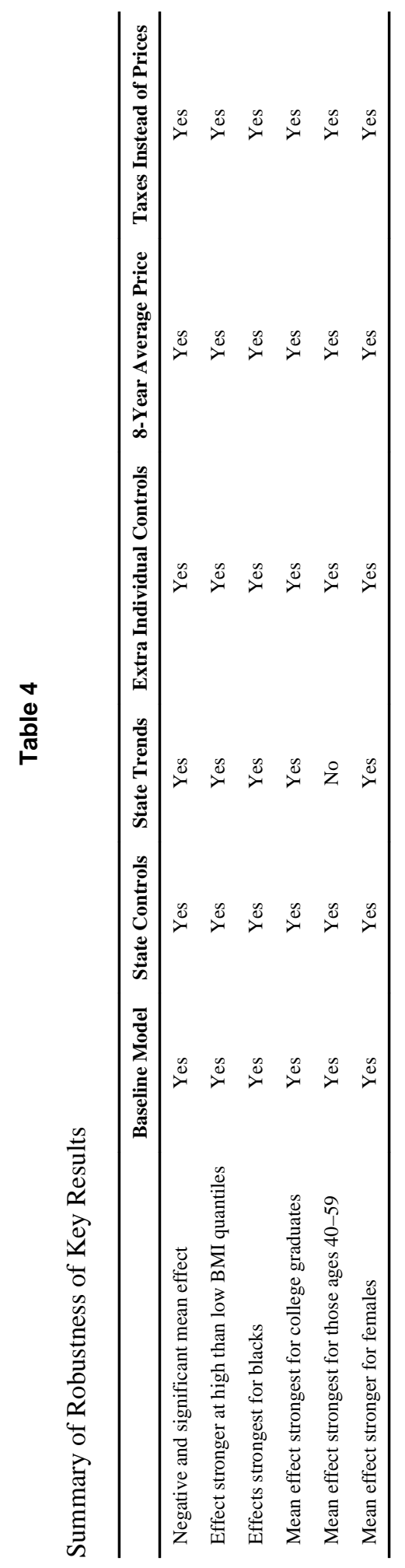

J Health Econ. Author manuscript; available in PMC 2013 September 01. 


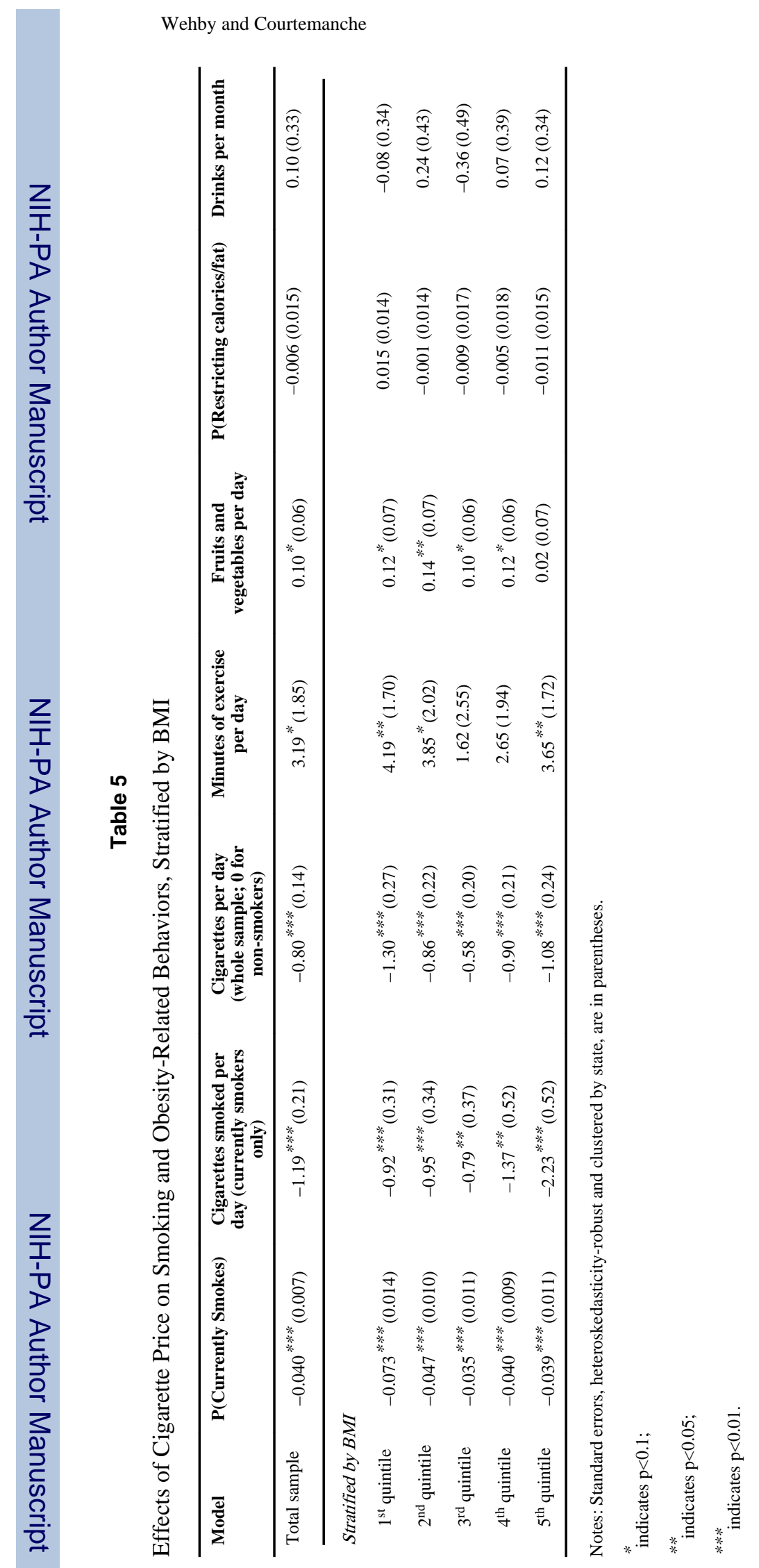

Page 23 